\title{
FLEXIBLE MEASURES IN PRODUCTION PROCESS: A DEA-BASED APPROACH
}

\author{
Alireza Amirteimoori ${ }^{1}$ And Ali Emrouznejad ${ }^{2}$
}

\begin{abstract}
Data envelopment analysis (DEA) has been proven as an excellent data-oriented efficiency analysis method for comparing decision making units $(D M U s)$ with multiple inputs and multiple outputs. In conventional DEA, it is assumed that the status of each measure is clearly known as either input or output. However, in some situations, a performance measure can play input role for some $D M U s$ and output role for others. Cook and Zhu [Eur. J. Oper. Res. 180 (2007) 692-699] referred to these variables as flexible measures. The paper proposes an alternative model in which each flexible measure is treated as either input or output variable to maximize the technical efficiency of the $D M U$ under evaluation. The main focus of this paper is on the impact that the flexible measures has on the definition of the PPS and the assessment of technical efficiency. An example in UK higher education intuitions shows applicability of the proposed approach.
\end{abstract}

Keywords. Data envelopment analysis, efficiency, flexible measure.

Mathematics Subject Classification. 90B030, 90B060.

\section{INTRODUCTION}

Data envelopment analysis (DEA) is concerned with comparative assessment of the efficiency of decision making units $(D M U s)$. In the classical DEA models, the efficiency of a $D M U$ is obtained by maximizing ratio of the weighted sum of its

Received December 17, 2010. Accepted April 15, 2011.

1 Department of Applied Mathematics, Islamic Azad University, Rasht branch, Rasht, Iran. teimoori@guilan.ac.ir

2 Operations \& Information Management Group, Aston Business School, Aston University,

Birmingham B4 7ET, UK. a.emrouznejad@aston.ac.uk 
outputs to the weighted sum of its inputs, subject to the condition that this ratio does not exceed one for any $D M U$. Since the pioneering work of Charnes et al. [4], DEA has demonstrated to be an effective technique for measuring the relative efficiency of a set of homogeneous DMUs which utilize the same inputs to produce the same outputs. DEA has been used in many contexts including education systems, health care units, agricultural productions, military logistics and many other applications (see Charnes et al. [5], Cooper et al. [10] and Emrouznejad et al. [11]). In conventional DEA applications, given a set of available measures, it is assumed that the status of each measure is clearly stated as an input or an output variable in the production process prior to using DEA. For example, in a conventional study of bank branch activities, one can clearly specify that staff is an input, and profit earned is an output. However, in higher education application, for instance, there is always a question that in assessing universities activities, whether a research income is an input or an output? In the literature, many authors suggested that it should be considered as an input, because it is the money earned by university and it is used in the same period. Others said that it is the income that universities earned so it should be considered as an output. However in the interest of gaining a higher efficiency score some universities may keep the research income in the input side and some other universities in the output side. The main question is "how to decide about the role of research income for each university?".

Cook and Bala [6] and Cook and Zhu [8] have pointed a very similar question in the context of DEA. Cook and Bala [6] examined the problem of deciding the appropriate status of flexible measures when additional information is present. Specifically, they investigate the situation where bank branch consultants provide additional "classification data" specifying quality of each branch; good versus poor branch. Their idea was to assign a status to each flexible measure such as to provide efficiency scores that are in best agreement with expert opinion. Cook and Zhu [8] considered variables whose status are flexible and proposed a different method for classifying these variables by introducing a fractional programming problem to accommodate flexible measures. Their model is later transformed to a linear form using the Charnes and Cooper [3] transformation procedure (see also [12]).

One main drawback in the model proposed by Cook and Bala [6] is the requirement to enter extra information to decide about the status of each variable and the main weakness in the model proposed by Cook and Zhu [8] is that their model overestimates the efficiency. The current paper, suggests a different approach in which each flexible measure of the model is treated as either input or output to maximize the technical efficiency of the $D M U$ under evaluation. The main focus of this paper is on the impact that the flexible measures have on the definition of the PPS and the assessment of technical efficiency.

The paper is organized as follows: Section 2 provides the basic DEA model. The third section introduces a DEA-based approach for modeling production processes in the presence of flexible measures. A comparison with the Cook and Zhu [8] model is given in Section 4. Section 5 illustrates the usefulness and applicability of the proposed model in assessing higher education institutions in UK. Conclusions and further remarks are given in Section 5. 


\section{DEA EFFICIENCY ANALYSIS}

To describe the DEA efficiency measurement, assume there are $n$ DMUs and the performance of each $D M U$ is characterized by a production process of $m$ inputs $\left(x_{i j}: i=1, \ldots, m\right)$ to yields $s$ outputs $\left(y_{r j}: r=1, \ldots, s\right)$. The ratio DEA model also known as the CCR model, measures the efficiency of $D M U_{o}$ by maximizing the ratio of its weighted sum of outputs to its weighted sum of inputs, i.e.

$$
\theta_{o}=\frac{\sum_{r=1}^{s} u_{r} y_{r o}}{\sum_{i=1}^{m} v_{i} x_{i o}}
$$

where the maximum is sought subject to the conditions that this ratio does not exceed one for any $D M U_{j}$ and all the input and output weights are positive. Hence the following fractional model should be solved to obtain the efficiency score of $D M U_{o}$ (Charnes et al. [4]):

$$
\begin{aligned}
& \operatorname{Max} \theta_{o}=\frac{\sum_{r=1}^{s} u_{r} y_{r o}}{\sum_{i=1}^{m} v_{i} x_{i o}} \\
& \text { s.t. } \frac{\sum_{r=1}^{s} u_{r} y_{r j}}{\sum_{i=1}^{m} v_{i} x_{i j}} \leqslant 1, \quad j=1, \ldots, n, \\
& \quad u_{r}, v_{i} \geqslant \varepsilon, \quad \text { for all } r, i,
\end{aligned}
$$

where $\varepsilon>0$ is a non-archimedean constraint. This linear fractional programming problem can be reduced to a linear programming model (2.2) by using Charnes and Cooper [3] transformation.

$$
\begin{aligned}
\operatorname{Max} & \theta_{o}=\sum_{r=1}^{s} \bar{u}_{r} y_{r o} \\
\text { s.t. } & \sum_{i=1}^{m} \bar{v}_{i} x_{i o}=1, \\
& \sum_{r=1}^{s} \bar{u}_{r} y_{r j}-\sum_{i=1}^{m} \bar{v}_{i} x_{i j} \leqslant 0, \quad j=1, \ldots, n, \\
& \bar{u}_{r}, \bar{v}_{i} \geqslant \bar{\varepsilon}, \quad \text { for all } r, i .
\end{aligned}
$$

This model is a constant returns to scale (CRS) program and it assumes that the status of all input/output variables are known prior to solving the model. The efficiency ratio $\theta_{o}$ ranges between zero and one, with $D M U_{o}$ being considered relatively efficient if it receives a score of one. From a managerial perspective, this model delivers assessments and targets with an output maximization orientation. 


\section{Flexible measures in production process}

\subsection{An AXIOMATIC FOUNDATION}

Suppose we have $n D M U s$, and that each $D M U_{j}: j=1, \ldots, n$ uses $m$ inputs $x_{i j}: i=1, \ldots, m$ to produce $s$ outputs $y_{r j}: r=1, \ldots, s$. Suppose also that there exist $t$ flexible measures $z_{k j}: k=1, \ldots, t$, whose input/output statuses are not known, some $D M U s$ may use these measures, or some of these measures, as inputs and other DMUs may use them as outputs. The unit under evaluation is denoted by $D M U_{o}:\left(x_{o}, y_{o}\right)$.

Without loss of generality assume there are only three variables; $x, y$ and $z$; in the assessment model. Let $T$ be the production possibility set of technology under consideration. We postulate the following:

A1 Feasibility of observed data. $\left(x_{j}, y_{j}, z_{j}\right) \in T$ for any $j=1,2, \ldots, n$.

A2 Unbounded ray. $(x, y, z) \in T$ implies $\alpha(x, y, z) \in T$ for any $\alpha \geqslant 0$.

A3 Convexity. Let $\left(x^{\prime}, y^{\prime}, z^{\prime}\right) \in T$ and $\left(x^{\prime \prime}, y^{\prime \prime}, z^{\prime \prime}\right) \in T$. Then, for any $\lambda \in[0,1]$ the unit $\lambda\left(x^{\prime}, y^{\prime}, z^{\prime}\right)+(1-\lambda)\left(x^{\prime \prime}, y^{\prime \prime}, z^{\prime \prime}\right) \in T$.

A4 Free disposability. $(x, y, z) \in T, x^{\prime} \geqslant x$ and $y^{\prime} \leqslant y$, and (either $z^{\prime} \geqslant$ $z$ or $\left.z^{\prime} \leqslant z\right)$, implies $\left(x^{\prime}, y^{\prime}, z^{\prime}\right) \in T$.

A5 Minimal extrapolation. For each $T$ satisfying in the axioms A1-A4, we have $T \subseteq T^{\prime}$.

It is noted that A5 is different with standard axioms presented in Banker et al. [1]. Now, an Algebraic representation of the PPS of the technology T, which satisfying the axioms $\mathrm{A} 1-\mathrm{A} 5$, is given.

Theorem 3.1. The PPS T, which satisfies the axioms A1-A5, is defined as

$$
\begin{aligned}
T= & \left\{(x, y, z): x \geqslant \sum_{j=1}^{n} \lambda_{j} x_{j}, y \leqslant \sum_{j=1}^{n} \lambda_{j} y_{j},\left(\text { either } z \leqslant \sum_{j=1}^{n} \lambda_{j} z_{j} \text { or } z \geqslant \sum_{j=1}^{n} \lambda_{j} z_{j}\right),\right. \\
& \left.\lambda_{j} \geqslant 0, \quad j=1,2, \ldots, n\right\} .
\end{aligned}
$$

Proof. It is obvious that the set $T$ satisfies A1-A4. To see that $T$ is the minimal set, assume that $T^{\prime}$ also satisfies A1-A4. We need to show that $(x, y, z) \in T$ implies that $(x, y, z) \in T^{\prime}$. Consider the following representation of the unit $(x$, $y, z)$.

$$
\begin{aligned}
x & \geqslant \sum_{j=1}^{n} \lambda_{j} x_{j} \\
y & \leqslant \sum_{j=1}^{n} \lambda_{j} y_{j} \\
\text { either } \quad z & \leqslant \sum_{j=1}^{n} \lambda_{j} z_{j} \\
\text { or } \quad z & \geqslant \sum_{j=1}^{n} \lambda_{j} z_{j} .
\end{aligned}
$$


For the vector $\lambda=\left(\lambda_{1}, \lambda_{2}, \ldots, \lambda_{n}\right)$ from this representation, define

$$
\left(x_{\lambda}, y_{\lambda}, z_{\lambda}\right)=\left(\sum_{j=1}^{n} \lambda_{j} x_{j}, \sum_{j=1}^{n} \lambda_{j} y_{j}, \sum_{j=1}^{n} \lambda_{j} z_{j}\right) .
$$

It is clear that $\left(x_{\lambda}, y_{\lambda}, z_{\lambda}\right) \in T^{\prime}$, and this unit dominates $(x, y, z)$ in the Pareto sense. So, we conclude that $(x, y, z) \in T^{\prime}$. This completes the proof.

\subsection{A DEA MOdEL With Flexible MeAsure: AN INPUt ORIENTED APPROACH}

Based on the proposed PPS and as results of Theorem 3.1 we propose the following model for measuring efficiency of $D M U_{o}$. This is an input oriented model, each $D M U$ will set the status of variable $z$ on the interest of their efficiency level.

Min $\theta$

s.t.

$$
\begin{aligned}
& \sum_{j=1}^{n} \lambda_{j} x_{j} \leqslant \theta x_{o}, \\
& \sum_{j=1}^{n} \lambda_{j} y_{j} \geqslant y_{o}, \\
& \left\{\begin{array}{l}
\text { either } \\
\sum_{j=1}^{n} \lambda_{j} z_{j} \leqslant \theta z_{o}, \\
\sum_{j=1}^{n} \lambda_{j} z_{j} \geqslant z_{o},
\end{array}\right. \\
& \lambda_{j} \geqslant 0, \quad j=1,2, \ldots, n .
\end{aligned}
$$

Obviously this is not a straightforward linear programming. Hence the following procedure explains the transformation of the above model to a mixed integer linear program.

It is to be noted that one and only one of the constraints (3.3) or (3.4) must be hold for variable $z$. Suppose $M$ be a large positive number. Consider the following constraints:

$$
\begin{gathered}
\sum_{j=1}^{n} \lambda_{j} z_{j} \leqslant \theta z_{o}+M \delta_{1} \\
-\sum_{j=1}^{n} \lambda_{j} z_{j} \leqslant-z_{o}+M \delta_{2} \\
\delta_{1}+\delta_{2}=1, \\
\delta_{1}, \delta_{2} \in\{0,1\} .
\end{gathered}
$$


Clearly selecting $\delta_{1}=0$ forces $\delta_{2}=1$, hence, the constrain (3.6) is redundant and (3.5) holds. This means that $z_{o}$ is selected as an input for $D M U_{o}$. On the other hand, if we let $\delta_{1}=1$, then $\delta_{2}=0$, hence (3.5) is redundant and (3.6) holds. In this case, $z_{o}$ is selected as an output for $D M U_{o}$. Model (3.1)-(3.4) can now be restated in the following mixed-integer linear program:

$\operatorname{Min} \theta$

$$
\begin{aligned}
& \text { s.t. } \\
& \sum_{j=1}^{n} \lambda_{j} x_{j} \leqslant \theta x_{o} \\
& \sum_{j=1}^{n} \lambda_{j} y_{j} \geqslant y_{o} \\
& \sum_{j=1}^{n} \lambda_{j} z_{j} \leqslant \theta z_{o}+M \delta_{1} \\
& \sum_{j=1}^{n} \lambda_{j} z_{j} \leqslant-z_{o}+M \delta_{2} \\
& \delta_{1}+\delta_{2}=1 \\
& \delta_{1}, \delta_{2} \in\{0,1\} \\
& \lambda_{j} \geqslant 0, \quad j=1,2, \ldots, n \text {. }
\end{aligned}
$$

\subsection{Generalization of the Proposed MOdel}

In model (3.9) we assumed there are only 3 variables; $x, y$ and $z$; where the status of variable $z$ to be determined by DEA model. Consider now that there are multi-inputs, $x_{i j}: i=1, \ldots, m$, multi-outputs, $y_{r j}: r=1, \ldots, s$, and multi-flexible measures, $z_{k j}: k=1, \ldots, t$. To generalize the proposed model we should allow each $D M U$ to select the status of each flexible measure, so each $D M U$ sets some of the flexible measures to input variables and some others to output variables to secure the best possible efficiency score. In this case model (3.10) is proposed.

$\operatorname{Min} \theta$

s.t.

$$
\begin{array}{ll}
\sum_{j=1}^{n} \lambda_{j} x_{i j} \leqslant \theta x_{i o}, & i=1,2, \ldots, m, \\
\sum_{j=1}^{n} \lambda_{j} y_{r j} \geqslant y_{r o}, & r=1,2, \ldots, s, \\
\sum_{j=1}^{n} \lambda_{j} z_{k j} \leqslant \theta z_{k o}+M \delta_{1 k}, & k=1,2, \ldots, t, \\
\sum_{j=1}^{n} \lambda_{j} z_{k j} \leqslant-z_{k o}+M \delta_{2 k}, & k=1,2, \ldots, t, \\
\delta_{1 k}+\delta_{2 k}=1, & k=1,2, \ldots, t \\
\delta_{1 k}, \delta_{2 k} \in\{0,1\}, & k=1,2, \ldots, t, \\
\lambda_{j} \geqslant 0, \quad j=1,2, \ldots, n . &
\end{array}
$$


The above model is an input oriented measure. A similar model, see model (3.11), can be formulated to present output orientation DEA with flexible measures.

$\operatorname{Max} \phi$

$$
\begin{aligned}
& \text { s.t. } \\
& \sum_{j=1}^{n} \lambda_{j} x_{i j} \leqslant x_{i o}, \quad i=1,2, \ldots, m, \\
& \sum_{j=1}^{n} \lambda_{j} y_{r j} \geqslant \phi y_{r o}, \quad r=1,2, \ldots, s, \\
& \sum_{j=1}^{n} \lambda_{j} z_{k j} \leqslant z_{k o}+M \delta_{1 k}, \quad k=1,2, \ldots, t, \\
& \sum_{j=1}^{n} \lambda_{j} z_{k j} \leqslant-\phi z_{k o}+M \delta_{2 k}, \quad k=1,2, \ldots, t, \\
& \delta_{1 k}+\delta_{2 k}=1 \text {, } \\
& \delta_{1 k}, \delta_{2 k} \in\{0,1\}, \quad k=1,2, \ldots, t, \\
& \lambda_{j} \geqslant 0, \quad j=1,2, \ldots, n \text {. }
\end{aligned}
$$

One important conclusion is that unlike standard DEA, even under constant returns to scale, the input-oriented model (3.10) and the output oriented model (3.11) may produce different efficiency scores. This is obviously expected under flexible measures since one $D M U$ may consider one of its flexible measures as an input variable in one model but an output variable in the other model.

\section{Comparison with the Cook and Zhu [8] model}

In this section, we compare the model developed in this paper with the following model proposed by Cook and Zhu [8].

$$
\begin{aligned}
\operatorname{Max} & \bar{e}_{o}=\sum_{r=1}^{s} \mu_{r} y_{r o}+\sum_{k=1}^{t} \delta_{k} z_{k o} \\
\text { s.t. } & \sum_{i=1}^{m} v_{i} x_{i o}+\sum_{k=1}^{t} \gamma_{k} z_{k o}-\sum_{k=1}^{t} \delta_{k} z_{k o}=1, \\
& \sum_{r=1}^{s} \mu_{r} y_{r j}+2 \sum_{k=1}^{t} \delta_{k} z_{k j}-\sum_{i=1}^{m} v_{i} x_{i j}-\sum_{k=1}^{t} \gamma_{k} z_{k j} \leqslant 0, j=1,2, \ldots, n, \\
& 0 \leqslant \delta_{k} \leqslant M d_{k}, \quad k=1,2, \ldots, t, \\
& \delta_{k} \leqslant \gamma_{k} \leqslant \delta_{k}+M\left(1-d_{k}\right), \quad k=1,2, \ldots, t, \\
& \mu_{r}, v_{i}, \gamma_{k}, \delta_{k} \geqslant 0, \quad \text { for all } i, r, k, \\
& d_{k} \in\{0,1\}, \quad k=1,2, \ldots, t .
\end{aligned}
$$

This is a mixed integer linear programming in which $D M U_{j}$ produces $s$ different outputs $y_{r j}: r=1, \ldots, s$ using $m$ different inputs $x_{i j}: i=1, \ldots, m$. Also, the values assumed by flexible measures are denoted as $z_{k j}: k=1, \ldots, t$. 
One main drawback from Cook and Zhu [8] model is that it is a very optimistic model and the following two theorems show that model (4.1) is always overestimating the efficiency.

First consider the following two models. Model (4.2) is a CCR model assuming all flexible measures as input variables and model (4.3) is a CCR model assuming all flexible measure as output variables.

$$
\begin{aligned}
\operatorname{Max} & \breve{e}_{o}=\sum_{r=1}^{s} \mu_{r} y_{r o} \\
\text { s.t. } & \sum_{i=1}^{m} v_{i} x_{i o}+\sum_{k=1}^{t} \gamma_{k} z_{k o}=1, \\
& \sum_{r=1}^{s} \mu_{r} y_{r j}-\sum_{i=1}^{m} v_{i} x_{i j}-\sum_{k=1}^{t} \gamma_{k} z_{k j} \leqslant 0, \quad j=1,2, \ldots, n, \\
& \mu_{r}, v_{i}, \gamma_{k} \geqslant 0, \quad \text { for all } i, r, k . \\
\text { Max } & \widehat{\mathrm{e}_{o}}=\sum_{r=1}^{s} \mu_{r} y_{r o}+\sum_{k=1}^{t} \delta_{k} z_{k o} \\
\text { s.t. } & \sum_{i=1}^{m} v_{i} x_{i o}=1, \\
& \sum_{r=1}^{s} \mu_{r} y_{r j}+\sum_{k=1}^{t} \delta_{k} z_{k j}-\sum_{i=1}^{m} v_{i} x_{i j} \leqslant 0, \quad j=1,2, \ldots, n, \\
& \mu_{r}, v_{i}, \delta_{k} \geqslant 0, \quad \text { for all } i, r, k .
\end{aligned}
$$

Theorem 4.1. The optimal objective value to (4.2) and (4.3) are not greater than the optimal objective value to (4.1).

Proof. We first show that $\breve{e}_{o} \leqslant \bar{e}_{o}$. Let $\left(\mu^{*}, v^{*}, \gamma^{*}\right)$ be an optimal solution to (4.2). It is easy to see that $\left(\mu^{*}, v^{*}, \gamma^{*}, \delta=0, d=0\right)$ is a feasible solution to (4.1), hence, we must have $\breve{e}_{o} \leqslant \bar{e}_{o}$. Similarly, we can show that $\widehat{\mathrm{e}}_{o} \leqslant \bar{e}_{o}$, in which $\widehat{\mathrm{e}}_{o}$ is the optimal objective value to (4.3).

Theorem 4.2. The optimal objective value to (3.10) is not greater than the optimal objective value to (4.1).

Proof. Consider the dual formulation of the continuous relaxation of (3.10) as follows:

$$
\begin{aligned}
\operatorname{Max} & e_{o}^{*}=\sum_{r=1}^{s} \mu_{r} y_{r o}+\sum_{k=1}^{t} \rho_{k} z_{k o}+\sum_{k=1}^{t} \pi_{k} \\
\text { s.t. } & \sum_{i=1}^{m} v_{i} x_{i o}+\sum_{k=1}^{t} \gamma_{k} z_{k o}=1, \\
& \sum_{r=1}^{s} \mu_{r} y_{r j}-\sum_{i=1}^{m} v_{i} x_{i j}-\sum_{k=1}^{t} \gamma_{k} z_{k j}+\sum_{k=1}^{t} \rho_{k} z_{k j} \leqslant 0, j=1,2, \ldots, n, \\
& \pi_{k}+M \gamma_{k} \leqslant 0, \quad k=1,2, \ldots, t, \\
& \pi_{k}+M \rho_{k} \leqslant 0, \quad k=1,2, \ldots, t \\
& \mu_{r}, v_{i}, \gamma_{k}, \rho_{k} \geqslant 0, \quad \text { for all } i, r, k .
\end{aligned}
$$


It suffices to show that $e_{o}^{*} \leqslant \bar{e}_{o}$, in which $\bar{e}_{o}$ and $e_{o}^{*}$ are the optimal objective values to (4.1) and (4.4), respectively. Let $\left(\mu^{*}, \rho^{*}, v^{*}, \gamma^{*}, \pi^{*}\right)$ be an optimal solution to (4.4). It is easy to see that $\left(\bar{\mu}=\mu^{*}, \bar{\rho}=\rho^{*}, \bar{v}=v^{*}, \bar{\gamma}=\gamma^{*}-\rho^{*}\right)$ is a feasible solution to (4.1), hence, we have

$$
\begin{aligned}
e_{o}^{*} & =\sum_{r=1}^{s} \mu_{r}^{*} y_{r o}+\sum_{k=1}^{t} \rho_{k}^{*} z_{k o}+\sum_{k=1}^{t} \pi_{k}^{*} \leqslant \sum_{r=1}^{s} \mu_{r}^{*} y_{r o}+\sum_{k=1}^{t} \rho_{k}^{*} z_{k o} \\
& =\sum_{r=1}^{s} \bar{\mu}_{r} y_{r o}+\sum_{k=1}^{t} \bar{\rho}_{k} z_{k o} \leqslant \bar{e}_{o} .
\end{aligned}
$$

This completes the proof.

\section{Illustration OF THE PROPOSED MODEL: AN APPLICATION IN UK HIGHER EDUCATION INSTITUTIONS}

This section illustrates the proposed model in assessing UK higher education institutions. We apply our model to the data used by Cook and Zhu [8] (see also Beasley [2], Cook and Zhu [7] and Cook et al. [9]). Two factors are selected as inputs: general expenditure $\left(x_{1}\right)$ and equipment expenditure $\left(x_{2}\right)$, and three factors as outputs: undergraduate students $\left(y_{1}\right)$, postgraduate research $\left(y_{2}\right)$ and postgraduate teaching $\left(y_{3}\right)$. The flexible measure here is the research income $\left(z_{1}\right)$. The status of research income for each university will be determined by the model. The data set consists of 50 universities as shown in appendix.

The results of model (3.10) are reported under heading "efficiency with flexible variable" in Table 1 . The optimal values to $\delta_{1}$ and $\delta_{2}$ indicate that either research income was considered as an input or as an output variable in the assessment model. As can be seen 25 (out of the 50) universities treat the research income as an output measure, and 25 universities treat it as an input measure in the model.

Cook and Zhu identified 21 efficient universities, our model found that there are only 7 efficient universities, over all we think that the Cook and Zhu model overestimated the efficiency across all other universities. Theoretically this was proved in the previous section. Table 1 clearly shows that the Cook and Zhu [8] efficiency is always higher than the efficiency obtained as compare to our model. Further, we investigated the efficiency of higher education institution in two cases when we used simple CCR model one with research income as an input and another model with research income as an output. These are also reported in Table 1. As an example take university 12 , where our efficiency is $58 \%$, the two efficiency when considering research income as input and as an output are $67 \%$ and $58 \%$ while the cook and Zhu efficiency is $71 \%$, it is well overestimated, it can be seen that it is outside the interval $[58 \%, 67 \%]$. As another example take university 26 , with efficiency of $43 \%$ and $57 \%$, respectively, when we use CCR model and consider research income as an output or as an input, respectively. We doubt that this university, which is within lowest efficient universities, become efficient, as calculated in Cook and Zhu, just by adjusting its research income. It is very clear 
TABLE 1. Efficiency assessment of higher education institutions, a comparative study.

\begin{tabular}{|c|c|c|c|c|c|c|c|}
\hline DMU & $\begin{array}{c}\text { Efficiency, } \\
\text { research income as } \\
\text { input variable }\end{array}$ & $\begin{array}{c}\text { Efficiency, } \\
\text { research income as } \\
\text { output variable }\end{array}$ & $\begin{array}{l}\text { Efficiency, } \\
\text { research income as } \\
\text { flexible variable }\end{array}$ & $\delta_{1}$ & $\delta_{2}$ & $\begin{array}{c}\text { Cook and } \\
\text { Zhu } \\
(2007) \\
\end{array}$ & d \\
\hline 1 & 1 & 1 & 1 & 1 & 0 & 1 & \begin{tabular}{|l|l|}
1 & \\
\end{tabular} \\
\hline 2 & 0.64 & 0.61 & 0.61 & 0 & 1 & 1 & 0 \\
\hline 3 & 0.66 & 0.84 & 0.66 & 1 & 0 & 0.84 & 0 \\
\hline 4 & 0.69 & 0.65 & 0.65 & 0 & 1 & 0.69 & 1 \\
\hline 5 & 0.89 & 1 & 0.89 & 1 & 0 & 1 & 0 \\
\hline 6 & 1 & 0.79 & 0.79 & 0 & 1 & 1 & 0 \\
\hline 7 & 1 & 1 & 1 & 1 & 0 & 1 & 1 \\
\hline 8 & 0.81 & 0.75 & 0.75 & 0 & 1 & 0.81 & 1 \\
\hline 9 & 0.53 & 1 & 0.53 & 1 & 0 & 1 & 0 \\
\hline 10 & 0.91 & 0.89 & 0.89 & 0 & 1 & 0.91 & 1 \\
\hline 11 & 0.75 & 0.89 & 0.75 & 1 & 0 & 0.89 & 0 \\
\hline 12 & 0.67 & 0.58 & 0.58 & 0 & 1 & 0.71 & 1 \\
\hline 13 & 0.77 & 0.80 & 0.77 & 1 & 0 & 0.80 & 0 \\
\hline 14 & 0.70 & 0.75 & 0.70 & 1 & 0 & 0.77 & 0 \\
\hline 15 & 0.69 & 0.70 & 0.69 & 1 & 0 & 0.70 & 0 \\
\hline 16 & 0.52 & 0.53 & 0.52 & 1 & 0 & 0.54 & 0 \\
\hline 17 & 0.82 & 0.52 & 0.52 & 0 & 1 & 0.82 & 1 \\
\hline 18 & 0.63 & 0.59 & 0.59 & 0 & 1 & 0.63 & 1 \\
\hline 19 & 1 & 1 & 1 & 1 & 0 & 1 & 1 \\
\hline 20 & 0.87 & 0.73 & 0.73 & 0 & 1 & 1 & 0 \\
\hline 21 & 0.62 & 0.59 & 0.59 & 0 & 1 & 0.70 & 0 \\
\hline 22 & 0.72 & 0.66 & 0.66 & 0 & 1 & 0.72 & 1 \\
\hline 23 & 0.55 & 0.60 & 0.55 & 1 & 0 & 0.62 & 0 \\
\hline 24 & 1 & 0.46 & 0.46 & 0 & 1 & 1 & 0 \\
\hline 25 & 1 & 0.95 & 0.95 & 0 & 1 & 1 & 1 \\
\hline 26 & 0.57 & 0.43 & 0.43 & 0 & 1 & 1 & 0 \\
\hline 27 & 0.78 & 0.70 & 0.70 & 0 & 1 & 0.86 & 1 \\
\hline 28 & 0.81 & 1 & 0.81 & 1 & 0 & 1 & 0 \\
\hline 29 & 0.83 & 0.78 & 0.78 & 0 & 1 & 0.83 & 1 \\
\hline 30 & 0.89 & 0.63 & 0.63 & 0 & 1 & 1 & 0 \\
\hline 31 & 0.78 & 0.73 & 0.73 & 0 & 1 & 0.78 & 1 \\
\hline 32 & 0.84 & 0.89 & 0.84 & 1 & 0 & 0.90 & 0 \\
\hline 33 & 1 & 1 & 1 & 1 & 0 & 1 & 1 \\
\hline 34 & 1 & 0.93 & 0.93 & 0 & 1 & 1 & 0 \\
\hline 35 & 1 & 1 & 1 & 1 & 0 & 1 & 1 \\
\hline 36 & 0.73 & 0.81 & 0.73 & 1 & 0 & 0.84 & 0 \\
\hline 37 & 0.83 & 0.78 & 0.78 & 0 & 1 & 0.83 & 1 \\
\hline 38 & 0.81 & 0.83 & 0.81 & 1 & 0 & 0.83 & 0 \\
\hline 39 & 0.67 & 0.62 & 0.62 & 0 & 1 & 0.79 & 0 \\
\hline 40 & 0.74 & 0.74 & 0.74 & 0 & 1 & 0.74 & 1 \\
\hline 41 & 1 & 1 & 1 & 1 & 0 & 1 & 1 \\
\hline 42 & 0.82 & 0.80 & 0.80 & 0 & 1 & 0.85 & 0 \\
\hline 43 & 0.64 & 0.92 & 0.64 & 1 & 0 & 0.92 & 0 \\
\hline 44 & 1 & 1 & 1 & 1 & 0 & 1 & 0 \\
\hline 45 & 0.89 & 0.89 & 0.88 & 0 & 1 & 1 & 0 \\
\hline 46 & 0.85 & 0.85 & 0.85 & 0 & 1 & 1 & 0 \\
\hline 47 & 0.69 & 0.65 & 0.69 & 1 & 0 & 0.69 & 1 \\
\hline 48 & 0.79 & 0.84 & 0.79 & 1 & 0 & 0.94 & 0 \\
\hline 49 & 0.47 & 0.80 & 0.47 & 1 & 0 & 1 & 0 \\
\hline 50 & 0.84 & 0.84 & 0.84 & 1 & 0 & 0.84 & 0 \\
\hline
\end{tabular}


that Cook and Zhu estimation is not correct, in fact that it might be due to nature of the model they proposed.

\section{CONCLUSION AND FURTHER REMARKS}

In this paper we developed a DEA model to calculate the technical efficiency of $D M U s$ with flexible measures. For these types of production systems, the conventional DEA model is modified to incorporate flexible measures. The proposed approach is potentially useful in many applications including efficiency assessment of manufacturing, health care systems, educational authorities where generally a variable could be considered as an input or as an output. The paper started with an axiomatic study of the proposed model. Finally an application in higher education used to show the usefulness of the model. Further research can be done to transform other DEA models, e.g. slack-based model, using similar concept.

\section{REFERENCES}

[1] R.D. Banker, A. Charnes and W.W. Cooper, Some Methods for Estimating Technical and Scale Inefficiencies in Data Envelopment Analysis. Manage. Sci. 30 (1984) 1078-1092.

[2] J. Beasley, Comparing university departments. Omega 8 (1990) 171-183.

[3] A. Charnes and W.W. Cooper, Programming with Linear Fractional Functions. Naval Research. Logistics Quarterly 9 (1962) 181-186.

[4] A. Charnes, W.W. Cooper and E. Rhodes, Measuring the Efficiency of Decision Making Units. Eur. J. Oper. Res. 2 (1978) 429-444.

[5] A. Charnes, W.W. Cooper, A. Lewin and L.M. Seiford, Data Envelopment Analysis: Theory, Methodology and Applications. Kluwer Academic Publishers, Boston (1994).

[6] W.D. Cook and K. Bala, Performance measurement with classification information: An enhanced additive DEA model. Omega 31 (2003) 439-450.

[7] W.D. Cook and J. Zhu, Building performance standards into DEA structures. IIE Transactions 37 (2005) 267-275.

[8] W.D. Cook and J. Zhu, Classifying inputs and outputs in DEA. Eur. J. Oper. Res. 180 (2007) 692-699.

[9] W. D. Cook, M. Hababou and H. Tuenter, Multi-component efficiency measurement and shared inputs in data envelopment analysis: An application to sales and service performance in bank branches. J. Prod. Anal. 14 (2000) 209-224.

[10] W.W. Cooper, L.M. Seiford and K. Tone, Introduction to data envelopment analysis and its uses. Springer Publisher (2006).

[11] A. Emrouznejad, G. Tavares and B. Parker, Evaluation of research in efficiency and productivity: A survey and analysis of the first 30 years of scholarly literature in DEA. SocioEcon. Plan. Sci. 42 (2008) 151-157.

[12] M. Toloo, On classifying inputs and outputs in DEA: A revised model. Eur. J. Oper. Res. 198 (2009) 358-360. 
Appendix. Data set for higher education institutions

\begin{tabular}{|c|c|c|c|c|c|c|}
\hline$D M U$ & $\begin{array}{c}\text { G. } \\
\text { expenditure }\end{array}$ & $\begin{array}{c}\text { E. } \\
\text { expenditure }\end{array}$ & $\begin{array}{c}\text { UG } \\
\text { students }\end{array}$ & $\begin{array}{c}\mathrm{PG} \\
\text { research }\end{array}$ & $\begin{array}{c}\mathrm{PG} \\
\text { teaching }\end{array}$ & $\begin{array}{c}\text { Research } \\
\text { income }\end{array}$ \\
\hline 1 & 528 & 64 & 145 & 26 & 0 & 254 \\
\hline 2 & 2605 & 301 & 381 & 70 & 16 & 1485 \\
\hline 3 & 304 & 23 & 44 & 6 & 3 & 45 \\
\hline 4 & 1620 & 485 & 287 & 48 & 0 & 940 \\
\hline 5 & 490 & 90 & 91 & 30 & 8 & 106 \\
\hline 6 & 2675 & 767 & 352 & 170 & 4 & 2967 \\
\hline 7 & 422 & 0 & 70 & 31 & 12 & 298 \\
\hline 8 & 986 & 126 & 203 & 32 & 0 & 776 \\
\hline 9 & 523 & 32 & 60 & 17 & 0 & 39 \\
\hline 10 & 585 & 87 & 80 & 44 & 17 & 353 \\
\hline 11 & 931 & 161 & 191 & 20 & 0 & 293 \\
\hline 12 & 1060 & 91 & 139 & 37 & 0 & 781 \\
\hline 13 & 500 & 109 & 104 & 19 & 0 & 215 \\
\hline 14 & 714 & 77 & 132 & 24 & 0 & 269 \\
\hline 15 & 923 & 121 & 135 & 41 & 10 & 392 \\
\hline 16 & 1267 & 128 & 169 & 31 & 0 & 546 \\
\hline 17 & 891 & 116 & 125 & 24 & 0 & 925 \\
\hline 18 & 1395 & 571 & 176 & 41 & 14 & 764 \\
\hline 19 & 990 & 83 & 28 & 93 & 36 & 615 \\
\hline 20 & 3512 & 267 & 511 & 176 & 23 & 3182 \\
\hline 21 & 1451 & 226 & 198 & 53 & 0 & 791 \\
\hline 22 & 1018 & 81 & 161 & 34 & 5 & 741 \\
\hline 23 & 1115 & 450 & 148 & 36 & 4 & 347 \\
\hline 24 & 2055 & 112 & 207 & 48 & 1 & 2945 \\
\hline 25 & 440 & 74 & 115 & 9 & 0 & 453 \\
\hline 26 & 3897 & 841 & 353 & 93 & 28 & 2331 \\
\hline 27 & 836 & 81 & 129 & 37 & 0 & 695 \\
\hline 28 & 1007 & 50 & 174 & 30 & 7 & 98 \\
\hline 29 & 1188 & 170 & 253 & 38 & 0 & 879 \\
\hline 30 & 4630 & 628 & 544 & 217 & 0 & 4838 \\
\hline 31 & 977 & 77 & 94 & 52 & 26 & 490 \\
\hline 32 & 829 & 61 & 128 & 42 & 17 & 291 \\
\hline 33 & 898 & 39 & 190 & 19 & 1 & 327 \\
\hline 34 & 901 & 131 & 168 & 59 & 9 & 956 \\
\hline 35 & 924 & 119 & 119 & 85 & 37 & 512 \\
\hline 36 & 1251 & 62 & 193 & 56 & 13 & 563 \\
\hline 37 & 1011 & 235 & 217 & 36 & 0 & 714 \\
\hline 38 & 732 & 94 & 151 & 26 & 3 & 297 \\
\hline 39 & 444 & 46 & 49 & 21 & 2 & 277 \\
\hline 40 & 308 & 28 & 57 & 7 & 0 & 154 \\
\hline 41 & 483 & 40 & 117 & 23 & 0 & 531 \\
\hline 42 & 515 & 68 & 79 & 30 & 7 & 305 \\
\hline 43 & 593 & 82 & 101 & 10 & 1 & 85 \\
\hline 44 & 570 & 26 & 71 & 31 & 20 & 130 \\
\hline 45 & 1317 & 123 & 293 & 40 & 1 & 1043 \\
\hline 46 & 2013 & 149 & 403 & 53 & 2 & 1523 \\
\hline 47 & 992 & 89 & 161 & 31 & 1 & 743 \\
\hline 48 & 1038 & 82 & 151 & 60 & 13 & 513 \\
\hline 49 & 206 & 1 & 16 & 6 & 0 & 72 \\
\hline 50 & 1193 & 95 & 240 & 32 & 0 & 485 \\
\hline
\end{tabular}

Source: Beasley [2] 